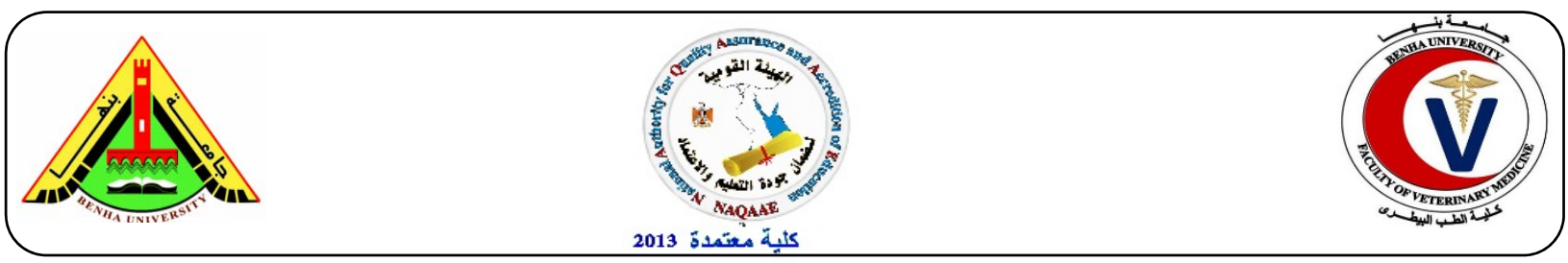

\title{
Molecular studies on antimicrobial resistance genes in salmonella isolated from poultry flocks in Egypt
}

\author{
Ashraf A. Abd El- Tawab a , Ahmed M. Ammar ${ }^{\text {b }}$, Soad A. Nasef ${ }^{c}$, Fatma I. El- Hofy and Nehal \\ M. Nabil ${ }^{\text {. }}$. \\ a Bacteriology, Immunology and Mycology Department, Faculty of Veterinary Medicine, Benha \\ University. ${ }^{b}$ Bacteriology, Mycology and Immunology Department, Faculty of Veterinary Medicine, \\ Zagzig University. ${ }^{c}$ Animal Health Research Institute, Dokki Lab., Giza. ${ }^{d}$ Animal Health Research \\ Institute, Dakahlia branch.
}

\begin{abstract}
A B S T R A C T
This study was aimed to investigate the wide spread resistance to different antimicrobial groups in between different Salmonellae isolated from poultry flocks in Egypt. A total of 63 Salmonella isolates (19 from chickens, 3 from ducks, 2 from turkeys, 2 from quails and 2 from pigeons) were examined in this study for their wide spread resistance against different antimicrobial agents. The antimicrobial susceptibility was applied on all isolates then PCR was applied for all resistant isolates to detect the resistance genes of different antimicrobial agents (qepA, qnrS, aac $\left(6^{\prime}\right)$ - ib-cr) for quinolone resistant isolates, bla Tem for $\beta$ lactam resistant isolates, sul1 for Sulfonamide resistant isolates, floR for Florphenicol resistant isolates, aadA2 for Streptomycin resistant isolates and tetA (A) for Oxytetracycline resistant isolates, in addition to gyrase enzyme gene (gyrA). DNA sequencing was done for three selected isolates to detect possible mutations in the quinolone-resistance determining regions of the gyrA gene.
\end{abstract}

Keywords: Salmonella, resistance genes, poultry

\section{INTRODUCTION}

$\mathrm{A}$ vian salmonellosis is a problem of economic concern to all phases of poultry industry from production to marketing. As a result of extensive use of antibiotics in human and veterinary medicine, serious increase in the spreading of multiple antibiotic resistant Salmonella has occurred (Cruchaga et al., 2001). Antimicrobial resistance is a global public health problem. Although all countries are affected, the extent of the problem in the developing nations is unknown (Ang et al., 2004). From the beginning of the 1990s, strains of Salmonella which are resistant to a range of antimicrobials, including firstchoice agents or the treatment of human and animals, have emerged and are threatening to become a serious public health problem. This resistance results from the use of antimicrobials both in human and animal husbandry (WHO, 2006). This study aimed to detect the different antimicrobial resistance genes in between different types of Salmonellae.

\section{MATERIAL AND METHODS}

\subsection{Source of Salmonella strains used in this study}

A total of 63 isolates were used in this study; 19 strains isolated from chickens ( $S$. Typhimurium, $S$. Apeyeme, $S$. Kentucky, $S$. Daula, $S$. Newport, $S$. Tamale, $S$. Molade, $S$. Colindale, $S$. Lexington, S. Bargny, $S$. 
Enteritidis, S. Papuana, S. Labadi, $S$. Santiago, S. Magherafelt, S. Rechovot, $S$. Takoradi, S. Angers, S. Shubra and untyped Salmonella), 3 strains isolated from ducks (S. Inganda, S. Infantis, S. Larochelle and untyped Salmonella), 2 strains isolated from turkeys ( $S$. Virchow and $S$. Vejle), 2 strains isolated from quails ( $S$. Shangani and $S$. Jedburgh) and 2 strains isolated from pigeons ( $S$. Alfort and $S$. Wingrove). The used isolates were collected from different poultry species in which they were isolated in Reference Laboratory for Veterinary Quality Control in Dokki.

\subsection{Antimicrobial susceptibility testing}

The antimicrobial susceptibility testing was done according to Finegold and Martin (1982) using the agar disc diffusion method on Mueller Hinton agar (Oxoid) plates. The used antimicrobial agents were Florphenicol $(30 \mu \mathrm{g})$, Nalidixic acid $(30 \mu \mathrm{g})$, Flumequine $(30 \mu \mathrm{g})$, Ciprofloxacin $(5 \mu \mathrm{g})$, Enrofloxacin $(5 \mu \mathrm{g})$, Norfloxacin $(10 \mu \mathrm{g})$, Levofloxacin $(5 \mu \mathrm{g})$, Amoxicillin $(10 \mu \mathrm{g})$, Ampicillin - sulbactam $(20 \mu \mathrm{g})$, Cefotaxime $(30 \mu \mathrm{g})$, Ceftriaxone $(30 \mu \mathrm{g})$, Ceftazidime (30 $\mathrm{g})$, Neomycin $(30 \mu \mathrm{g})$, Amikacin $(30 \mu \mathrm{g})$, Gentamycin $(10 \mu \mathrm{g})$, Streptomycin $(10 \mu \mathrm{g})$, Oxytetracyclin $(30 \mu \mathrm{g})$ and SulfamrthoxazoleTrimethoprim $(25 \mu \mathrm{g})$. The zones of inhibition that formed were measured to assess resistance or susceptibility according to the interpretation criteria established by (CLSI, 2011).

\subsection{3. Detection of resistance genes in isolated Salmonellae using PCR}

DNA was extracted from the isolates that showed resistance in antimicrobial susceptibility tests. The isolates that showed resistance to quinolones were examined for presence of [qepA, qnrS and aac(6')-ib-cr] genes while the isolates that showed resistance to Sulfamethoxazol/ Trimethoprim were examined for presence of sul 1 gene but the isolates that showed resistance to B- lactams were examined for the presence of bla TEM gene. The isolates that showed resistance to Florfenicol were examined for the presence of floR gene while the isolates that showed resistance to Streptomycin were examined for presence of aadA2 gene but the isolates that showed resistance to Oxytetracyclin were examined for tetA (A) gene. The DNA extraction for the selected isolates was performed using ABIOpure Genomic DNA extraction kit. The Oligonucleotide Primers which provided from Metabion (Germany) are listed in table (1). The primers were utilized in a $25 \mu 1$ reaction containing $12.5 \mu \mathrm{l}$ of EmeraldAmp Max PCR Master Mix (Takara, Japan), $1 \mu 1$ of each primer of $20 \mathrm{pmol}$ concentrations, 4.5 $\mu \mathrm{l}$ of water, and $6 \mu \mathrm{l}$ of template. The reaction was performed in a Biometra thermal cycler. The products of PCR were separated by electrophoresis on $1-1.5 \%$ agarose gel (ABgene) in 1x TBE buffer at room temperature. For gel analysis, $15 \mu \mathrm{l}$ of the products was loaded in each gel slot. A 100 bp DNA Ladder (Qiagen, USA) was used to determine the fragment sizes. The gel was photographed by a gel documentation system and the data was analyzed through computer software.

\subsection{Sequencing of the gyrA gene}

DNA sequencing for gyrA gene was done for 3 representative strains ( $S$. Infantis from ducks, $S$. Enteritidis from chickens and $S$. Vejle from turkeys). PCR products were purified using QIA quick PCR Product extraction kit (QIAGEN Inc, Valencia, CA, USA). Big dye Terminator V3.1 cycle sequencing kit. (Perkin-Elmer/Applied Biosystems, Foster City, CA) was used for the sequence reaction and then it was purified using Centrisep spin column. DNA sequences were obtained by Applied Biosystems 3130 genetic analyzer (HITACHI, Japan) and the phylogenetic tree was created by the MegAlign module of Lasergene DNA Star software.

\section{RESULTS}

\subsection{Results of the antimicrobial susceptibility tests for the isolated Salmonellae}


Fifty five isolates from a total of 63 Salmonella isolates showed the highest percentage of resistance $(87.3 \%)$ to Nalidixic acid and Flumequin. Five isolates from these 63 Salmonella isolates showed the lowest percentage of resistance $(7.9 \%)$ to Levofloxacin. Amikacin showed highest sensitivity $(100 \%)$ in all isolates from all examined species but 8 isolates showed the lowest sensitivity (12.7\%) to Nalidixic acid and Flumequin (Table 2) and (Table 3).

\subsection{Results of Polymerase Chain Reaction technique for different resistance genes in the examined isolates.}

All Salmonella isolates that showed resistance against different antimicrobial agents were examined by PCR to detect the incidence of resistance genes in the examined isolates which was (87\%) for sul
1, $(83.7 \%)$ for tetA (A), $(77.8 \%)$ for floR, (69.4\%) for bla TEM, (53.1\%) for aadA2, (19.6\%) for aac $\left(6^{\prime}\right)$-ib-cr, $(10.7 \%)$ for $q n r S$ and $(3.5 \%)$ for qepA (Table, 4$)$.

\subsection{DNA sequencing}

The 3 gyrA sequenced strains ( $S$. Infantis, $S$. Enteritidis and $S$. Vejle; isolates number 14 , 40,45 respectively in this study) showed two sits of mutation in the amino acids of $S$. Enteritidis (isolate NO. 40) (KP290113) at the position 87 which changed from Aspartic acid (D) (standard protein) to Glycine $(\mathrm{G})$ (mutated protein) and at the position 176 mutated from Valine (V) (standard protein) to Alanine (A) (mutated protein). No mutations in gyrA gene of $S$. Infantis (KP290114) or $S$. Vejle (KP290112) were recorded in this study (Table, 5).

Table 1. Oligonucleotide primers for PCR.

\begin{tabular}{|c|c|c|}
\hline Primers & Nucleotide Sequence & References \\
\hline $\begin{array}{c}\text { tetA (A) } \\
\text { (Tetracycline) }\end{array}$ & $\begin{array}{l}\text { F(5'-GGTTCACTCGAACGACGTCA -3') } \\
\text { R(5'-CTGTCCGACAAGTTGCATGA -3') }\end{array}$ & $\begin{array}{l}\text { Randall } \\
\text { et al., } 2004\end{array}$ \\
\hline $\begin{array}{c}\text { aadA2 } \\
\text { (Streptomycin) } \\
\text { blaTEM } \\
\text { (B-lactamas) }\end{array}$ & $\begin{array}{l}\text { F (5'-TGTTGGTTACTGTGGCCGTA -3') } \\
\text { R(5'-GATCTCGCCTTTCACAAAGC -3') } \\
\text { F(5'-ATCAGCAATAAACCAGC -3') } \\
\text { R(5'-CCCCGAAGAACGTTTTC -3') }\end{array}$ & $\begin{array}{l}\text { Walker et } \\
\text { al., } 2001 \\
\text { Colom et } \\
\text { al., } 2003\end{array}$ \\
\hline $\begin{array}{c}\text { sul } 1 \\
\text { (Sulfonamides) }\end{array}$ & $\begin{array}{l}\text { F(5'- CGGCGTGGGCTACCTGAACG-3') } \\
\text { R(5'- GCCGATCGCGTGAAGTTCCG-3') }\end{array}$ & $\begin{array}{l}\text { Sabarinath et } \\
\text { al., } 2011\end{array}$ \\
\hline $\begin{array}{c}\text { qepA } \\
\text { (Quinolones) }\end{array}$ & $\begin{array}{l}\text { F(5'-CGTGTTGCTGGAGTTCTTC -3') } \\
\text { R(5'-CTGCAGGTACTGCGTCATG -3') }\end{array}$ & $\begin{array}{l}\text { Cattoir } \\
\text { al., } 2008\end{array}$ \\
\hline $\begin{array}{l}\operatorname{aac}\left(6^{\prime}\right)-i b-c r \\
\text { (Quinolones) }\end{array}$ & $\begin{array}{l}\text { F(5'-CCCGCTTTCTCGTAGCA -3') } \\
\text { R(5'-TTAGGCATCACTGCGTCTTC -3') }\end{array}$ & $\begin{array}{l}\text { Lunn } \\
\text { al., } 2010\end{array}$ \\
\hline $\begin{array}{c}\text { qnrS } \\
\text { (Quinolones) }\end{array}$ & $\begin{array}{l}\text { F(5'-ACGACATTCGTCAACTGCAA -3') } \\
\text { R(5'-TAAATTGGCACCCTGTAGGC - -3') }\end{array}$ & $\begin{array}{l}\text { Robicsek et } \\
\text { al., } 2006\end{array}$ \\
\hline $\begin{array}{c}\text { floR } \\
\text { (Florphenicol) }\end{array}$ & $\begin{array}{l}\text { F(5'-TTTGGWCCGCTMTCRGAC -3') } \\
\text { R(5'-SGAGAARAAGACGAAGAAG -3') }\end{array}$ & $\begin{array}{l}\text { Doublet et } \\
\text { al., } 2003\end{array}$ \\
\hline $\begin{array}{c}\text { gyrA } \\
\text { (Quinolones } \\
\text { mutation gene) }\end{array}$ & $\begin{array}{l}\text { F (5'-AAATCTGCCCGTGTCGTTGGT-3') } \\
\text { R(5'-GCCATACCTACTGCGATACC-3') }\end{array}$ & $\begin{array}{l}\text { Fàbrega et } \\
\text { al., } 2009\end{array}$ \\
\hline
\end{tabular}


Table 2. Numbers and percentages of Salmonella isolates from poultry exhibiting resistance to various antimicrobials.

\begin{tabular}{|c|c|c|c|c|c|c|c|c|c|c|c|c|}
\hline \multirow[t]{2}{*}{$\begin{array}{l}\text { Antimicrobial } \\
\text { agent }\end{array}$} & \multicolumn{2}{|c|}{$\begin{array}{l}\text { resistant } \\
\text { chicken } \\
\text { isolates }\end{array}$} & \multicolumn{2}{|c|}{$\begin{array}{l}\text { resistant } \\
\text { duck } \\
\text { isolates }\end{array}$} & \multicolumn{2}{|c|}{$\begin{array}{c}\text { resistant } \\
\text { turkey } \\
\text { isolates }\end{array}$} & \multicolumn{2}{|c|}{$\begin{array}{l}\text { resistant } \\
\text { quail } \\
\text { isolates }\end{array}$} & \multicolumn{2}{|c|}{$\begin{array}{l}\text { resistant } \\
\text { pigeon } \\
\text { isolates }\end{array}$} & \multicolumn{2}{|c|}{ Total } \\
\hline & No & $\% *$ & No & $\%$ & No & $\%$ & No & $\%$ & No & $\%$ & No & $\% * *$ \\
\hline Florphenicol & 19 & 44.2 & 4 & 40 & 1 & 33.3 & 3 & 60 & 0 & 0 & 27 & 42.9 \\
\hline Nalidixic acid & 43 & 100 & 9 & 90 & 1 & 33.3 & 1 & 20 & 1 & 50 & 55 & 87.3 \\
\hline Flumequin & 43 & 100 & 9 & 90 & 1 & 33.3 & 1 & 20 & 1 & 50 & 55 & 87.3 \\
\hline Ciprofloxacin & 15 & 34.9 & 0 & 0 & 0 & 0 & 0 & 0 & 0 & 0 & 15 & 23.8 \\
\hline Enerofloxacin & 23 & 53.2 & 2 & 20 & 0 & 0 & 0 & 0 & 0 & 0 & 25 & 39.7 \\
\hline Norfloxacin & 9 & 20.9 & 1 & 10 & 0 & 0 & 0 & 0 & 0 & 0 & 10 & 15.9 \\
\hline Levofloxacin & 5 & 11.6 & 0 & 0 & 0 & 0 & 0 & 0 & 0 & 0 & 5 & 7.9 \\
\hline Amoxicillin & 39 & 90.7 & 5 & 50 & 1 & 33.3 & 2 & 40 & 2 & 100 & 49 & 77.8 \\
\hline Ampicilliln/sulbctam & 33 & 76.7 & 6 & 60 & 0 & 0 & 2 & 40 & 2 & 100 & 43 & 68.3 \\
\hline Cefotaxime & 7 & 16.3 & 0 & 0 & 0 & 0 & 0 & 0 & 0 & 0 & 7 & 11.1 \\
\hline Ceftriaxone & 10 & 23.3 & 1 & 10 & 0 & 0 & 0 & 0 & 0 & 0 & 11 & 17.5 \\
\hline Ceftazidime & 11 & 25.6 & 1 & 10 & 0 & 0 & 0 & 0 & 0 & 0 & 12 & 19.1 \\
\hline Neomycin & 18 & 41.9 & 4 & 40 & 1 & 33.3 & 0 & 0 & 0 & 0 & 23 & 36.5 \\
\hline Amikacin & 0 & 0 & 0 & 0 & 0 & 0 & 0 & 0 & 0 & 0 & 0 & 0 \\
\hline Gentamycin & 13 & 30.2 & 1 & 10 & 0 & 0 & 0 & 0 & 0 & 0 & 14 & 22.2 \\
\hline Streptomycin & 23 & 53.5 & 6 & 60 & 1 & 33.3 & 2 & 40 & 0 & 0 & 32 & 50.8 \\
\hline Oxytetracyclin & 34 & 79.1 & 6 & 60 & 1 & 33.3 & 1 & 20 & 1 & 50 & 43 & 68.3 \\
\hline Sulfa+trimethoprim & 25 & 58.1 & 4 & 40 & 1 & 33.3 & 1 & 20 & 0 & 0 & 31 & 49.2 \\
\hline
\end{tabular}

* The percentage calculated by dividing the number of isolates that showed resistance to each antimicrobial agent individually on the number of Salmonella isolates from each species $(19 / 43 \times 100=$ $44.2 \%)$.** The percentage calculated by dividing the total number of isolates that showed resistance from all species to each antimicrobial agent individually on the total number of Salmonella isolates from all species $(27 / 63 \times 100=42.9 \%)$.

\section{DISCUSSION}

According to the results concerning antimicrobial susceptibility tests in table (2)\& (3), 55 isolates showed the highest percentage of resistance $(87.3 \%)$ to Nalidixic acid and Flumequin. Five isolates showed the lowest percentage of resistance $(7.9 \%)$ to Levofloxacin. All Salmonella isolates from all examined species were sensitive to Amikacin (100\%) but 8 isolates from all examined isolates showed the lowest sensitivity $(12.7 \%)$ to Nalidixic acid and Flumequin. The obtained results were differed from Boris et al., (2012) who reported that all Salmonella isolates were sensitive to Chloramphenicol and Streptomycin $(100 \%)$ while 92 isolates $(58 \%)$ were sensitive to Nalidixic acid and of $66(41.7 \%)$ isolates were sensitive to all antibiotics. Contrary to these results Cardoso et al., (2006) who reported that Salmonella showed sensitivity to doxycycline hydrochloride with $100 \%$. However Khan et al., (2010) stated that all Salmonella isolates exhibit (100\%) resistant to Cephalexin and Rifampicin while about $90 \%$ and $88 \%$ of the isolates were resistant to Ampicillin and tetracycline. In this study forty three chicken isolates showed resistance with $100 \%$ against Nalidixic acid and Flumequin but five isolates showed the lowest resistance (11.6\%) to levofloxacin while 43 chicken isolates were sensitive to Amikacin (100\%) but 38 isolates were sensitive to Levofloxacin with a percentage 
Table 3. Numbers and percentages of sensitive Salmonella isolates from poultry.

\begin{tabular}{|c|c|c|c|c|c|c|c|c|c|c|c|c|}
\hline \multirow[t]{2}{*}{$\begin{array}{l}\text { Antimicrobial } \\
\text { agent }\end{array}$} & \multicolumn{2}{|c|}{$\begin{array}{l}\text { sensitive } \\
\text { chicken } \\
\text { isolates }\end{array}$} & \multicolumn{2}{|c|}{$\begin{array}{l}\text { sensitive } \\
\text { ducks } \\
\text { isolates }\end{array}$} & \multicolumn{2}{|c|}{$\begin{array}{l}\text { sensitive } \\
\text { turkeys } \\
\text { isolates }\end{array}$} & \multicolumn{2}{|c|}{$\begin{array}{l}\text { sensitive } \\
\text { quails } \\
\text { isolates }\end{array}$} & \multicolumn{2}{|c|}{$\begin{array}{l}\text { sensitive } \\
\text { pigeons } \\
\text { isolates }\end{array}$} & \multicolumn{2}{|c|}{ Total } \\
\hline & No & $\% *$ & No & $\%$ & No & $\%$ & No & $\%$ & No & $\%$ & No & $\% * *$ \\
\hline Florphenicol & 24 & 55.8 & 6 & 60 & 2 & 66.7 & 2 & 40 & 2 & 100 & 36 & 57.1 \\
\hline Nalidixic acid & 0 & 0 & 1 & 10 & 2 & 66.7 & 4 & 80 & 1 & 50 & 8 & 12.7 \\
\hline Flumequin & 0 & 0 & 1 & 10 & 2 & 66.7 & 4 & 80 & 1 & 50 & 8 & 12.7 \\
\hline Ciprofloxacin & 28 & 65.1 & 10 & 100 & 3 & 100 & 5 & 100 & 2 & 100 & 48 & 76.2 \\
\hline Enerofloxacin & 20 & 46.5 & 8 & 80 & 3 & 100 & 5 & 100 & 2 & 100 & 38 & 60.3 \\
\hline Norfloxacin & 34 & 79.1 & 9 & 90 & 3 & 100 & 5 & 100 & 2 & 100 & 53 & 84.1 \\
\hline Levofloxacin & 38 & 88.4 & 10 & 100 & 3 & 100 & 5 & 100 & 2 & 100 & 58 & 92.1 \\
\hline Amoxicillin & 4 & 9.3 & 5 & 50 & 2 & 66.7 & 3 & 60 & 0 & 0 & 14 & 22.2 \\
\hline Ampicilliln/sulbctam & 10 & 23.3 & 4 & 40 & 3 & 100 & 3 & 60 & 0 & 0 & 20 & 31.7 \\
\hline Cefotaxime & 36 & 83.7 & 10 & 100 & 3 & 100 & 5 & 100 & 2 & 100 & 56 & 88.9 \\
\hline Ceftriaxone & 33 & 52.4 & 9 & 90 & 3 & 100 & 5 & 100 & 2 & 100 & 52 & 82.5 \\
\hline Ceftazidime & 32 & 74.4 & 9 & 90 & 3 & 100 & 5 & 100 & 2 & 100 & 51 & 80.9 \\
\hline Neomycin & 25 & 58.1 & 6 & 60 & 2 & 66.7 & 5 & 100 & 2 & 100 & 40 & 63.5 \\
\hline Amikacin & 43 & 100 & 10 & 100 & 3 & 100 & 5 & 100 & 2 & 100 & 63 & 100 \\
\hline Gentamycin & 30 & 69.8 & 9 & 90 & 3 & 100 & 5 & 100 & 2 & 100 & 49 & 77.8 \\
\hline Streptomycin & 20 & 46.5 & 4 & 40 & 2 & 66.7 & 3 & 60 & 2 & 100 & 31 & 49.2 \\
\hline Oxytetracyclin & 9 & 20.9 & 4 & 40 & 2 & 66.7 & 4 & 80 & 1 & 50 & 20 & 31.7 \\
\hline Sulfa+trimethoprim & 18 & 41.9 & 6 & 60 & 2 & 66.7 & 4 & 80 & 2 & 100 & 32 & 50.8 \\
\hline
\end{tabular}

* The percentage calculated by dividing the number of isolates that were sensitive to each antimicrobial agent individually on the number of Salmonella isolates from each species $(24 / 43 \times 100=55.8 \%)$. ** The percentage calculated by dividing the total number of isolates that were sensitive to each antimicrobial agent individually (from all species) on the total number of Salmonella isolates from all species $(36 / 63 \times 100=57.1 \%)$.

$(88.4 \%)$ and 4 isolates were sensitive to Amoxicillin giving the lowest percentage $(9.3 \%)$ and these results agreed with Snow et al., (2011) who reported that all Salmonella isolates from commercial layer flocks in UK were sensitive to Amikacin with (100\%). The results in this study differ from Zdragas et al., (2012) who reported (5\%) of 23 resistance to Streptomycin (highest resistance rates) and $(2 \%)$ to Tetracycline and Nalidixic acid (lowest rate), Munawwar et al., (2010) who reported $(100 \%)$ resistance to Cephalexin and Rifampicin in Salmonella isolated from chicken meats in Dubai, while $87.88 \%$ of these Salmonellae were sensitive to ciprofloxacin and amikacin. Nine duck isolates in this study showed the highest resistance with (90\%) to Nalidixic acid and Flumequin while Norfloxacin, Ceftriaxone,
Ceftazidime and Gentamycin came in lowest percentage with (10\%).Ciprofloxacin, Levofloxacin, Cefotaxime and Amikacin showed (100\%) sensitivity while Nalidixic acid, Flumequin showed the lowest sensitivity with a percentage of $(10 \%)$. These results agreed with Rahman et al., (1999) who detected that $S$. Enteritidis was susceptible to Cefotaxime and Ciprofloxacin, Pan et al., (2009) who reported that Salmonella displayed a high level of resistance to Ampicillin, Streptomycin and Tetracycline. While these results differ from Hegazy (1991) who reported that 19 Salmonellae isolated from ducks in Kafr El- Sheik governorate were resistant to Ampcillin and Cephaloxin with percentage of $100 \%$ while Flumequine and Enrofloxacin were highly sensitive. 
Table 4. Results of Polymerase Chain Reaction technique for different resistance genes from the examined isolates.

\begin{tabular}{|c|c|c|c|c|c|c|c|c|c|c|}
\hline No & serotype & source & qерА & $q n r S$ & $\begin{array}{c}\operatorname{aac}\left(6^{\prime}\right)- \\
\text { ib-cr }\end{array}$ & $\begin{array}{c}\text { sul } \\
1 \\
\end{array}$ & $\begin{array}{c}\text { bla } \\
\text { TEM }\end{array}$ & floR & $\begin{array}{l}\text { aad } \\
\mathrm{A} 2\end{array}$ & $\begin{array}{l}\text { tetA } \\
\text { (A) }\end{array}$ \\
\hline 1 & S. Alfort & pigeon & - & - & - & $\mathrm{NE}$ & - & NE & $\mathrm{NE}$ & + \\
\hline 2 & S.Typhmurium & chicken & - & - & - & $\mathrm{NE}$ & NE & NE & NE & $\mathrm{NE}$ \\
\hline 3 & S.Typhmurium & chicken & - & - & + & $\mathrm{NE}$ & + & $\mathrm{NE}$ & - & - \\
\hline 4 & S.Virchow & turkey & $\mathrm{NE}$ & $\mathrm{NE}$ & $\mathrm{NE}$ & $\mathrm{NE}$ & $\mathrm{NE}$ & $\mathrm{NE}$ & $\mathrm{NE}$ & $\mathrm{NE}$ \\
\hline 5 & Untyped & chicken & - & - & + & $\mathrm{NE}$ & + & $\mathrm{NE}$ & $\mathrm{NE}$ & + \\
\hline 6 & S.Typhmurium & chicken & - & - & - & $\mathrm{NE}$ & + & + & + & + \\
\hline 7 & S. Apeyeme & chicken & - & - & - & $\mathrm{NE}$ & + & $\mathrm{NE}$ & + & + \\
\hline 8 & S.Typhmurium & chicken & - & - & - & $\mathrm{NE}$ & + & $\mathrm{NE}$ & + & + \\
\hline 9 & S.Typhmurium & chicken & - & - & - & + & + & NE & + & + \\
\hline 10 & S.Typhmurium & chicken & - & - & - & + & NE & NE & $\mathrm{NE}$ & + \\
\hline 11 & S. Kentucky & chicken & - & - & - & + & + & + & + & + \\
\hline 12 & S. Daula & chicken & - & - & - & $\mathrm{NE}$ & + & NE & NE & + \\
\hline 13 & S. Newport & chicken & - & - & - & + & + & $\mathrm{NE}$ & $\mathrm{NE}$ & + \\
\hline 14 & S. Infantis & duck & - & + & - & + & + & + & + & + \\
\hline 15 & S. Shangani & quail & - & - & - & $\mathrm{NE}$ & + & + & + & $\mathrm{NE}$ \\
\hline 16 & S. Tamale & chicken & - & + & - & $\mathrm{NE}$ & + & NE & + & + \\
\hline 17 & S. Molade & chicken & - & - & - & + & + & $\mathrm{NE}$ & $\mathrm{NE}$ & + \\
\hline 18 & S.Typhmurium & chicken & - & - & - & + & + & + & + & + \\
\hline 19 & Untyped & duck & - & + & - & + & - & + & - & + \\
\hline 20 & S. Newport & chicken & - & - & - & + & + & + & + & + \\
\hline 21 & S. Colindale & chicken & - & - & - & + & - & $\mathrm{NE}$ & - & + \\
\hline 22 & Untyped & duck & - & - & - & $\mathrm{NE}$ & $\mathrm{NE}$ & $\mathrm{NE}$ & - & - \\
\hline 23 & S. Shangani & quail & NE & $\mathrm{NE}$ & $\mathrm{NE}$ & $\mathrm{NE}$ & NE & + & + & $\mathrm{NE}$ \\
\hline 24 & S. Newport & chicken & - & - & - & + & - & + & + & + \\
\hline 25 & S.Virchow & turkey & $\mathrm{NE}$ & NE & NE & $\mathrm{NE}$ & $\mathrm{NE}$ & $\mathrm{NE}$ & $\mathrm{NE}$ & $\mathrm{NE}$ \\
\hline 26 & S. lexington & chicken & - & - & - & - & - & $\mathrm{NE}$ & - & + \\
\hline 27 & S. Bargny & chicken & - & - & + & NE & - & NE & - & + \\
\hline 28 & S. Rechovot & chicken & - & + & + & + & + & + & + & $\mathrm{NE}$ \\
\hline 29 & Untyped & duck & - & - & - & $\mathrm{NE}$ & $\mathrm{NE}$ & NE & NE & $\mathrm{NE}$ \\
\hline 30 & S. Magherafelt & chicken & - & - & - & $\mathrm{NE}$ & + & NE & NE & - \\
\hline 31 & S. Enteritidis & chicken & - & - & - & NE & - & + & NE & - \\
\hline 32 & S. Inganda & duck & - & - & - & NE & - & $\mathrm{NE}$ & - & + \\
\hline 33 & S. Magherafelt & chicken & - & + & - & + & + & NE & NE & + \\
\hline 34 & S.Typhmurium & chicken & - & - & - & + & + & - & $\mathrm{NE}$ & $\mathrm{NE}$ \\
\hline 35 & S. Santiago & chicken & - & - & - & $\mathrm{NE}$ & + & + & $\mathrm{NE}$ & + \\
\hline 36 & S. Infantis & duck & - & - & - & $\mathrm{NE}$ & + & NE & NE & $\mathrm{NE}$ \\
\hline 37 & S. Labadi & chicken & - & - & - & NE & - & - & - & + \\
\hline 38 & S. Molade & chicken & - & - & + & + & + & NE & - & + \\
\hline 39 & S. Tamale & chicken & - & - & + & $\mathrm{NE}$ & + & $\mathrm{NE}$ & $\mathrm{NE}$ & + \\
\hline
\end{tabular}


Table 4. (continued) Results of Polymerase Chain Reaction technique for different resistance genes from the examined isolates.

\begin{tabular}{lcccccccccc}
\hline No & serotype & source & qepA & qnrS & $\begin{array}{c}\text { aac(6')- } \\
\text { ib-cr }\end{array}$ & $\begin{array}{c}\text { sul } \\
1\end{array}$ & $\begin{array}{c}\text { bla } \\
\text { TEM }\end{array}$ & $\begin{array}{c}\text { floR } \\
\text { aad } \\
\text { A2 }\end{array}$ & $\begin{array}{c}\text { tetA } \\
\text { (A) }\end{array}$ \\
\hline 40 & S.Enteritidis & chicken & - & - & + & + & $\mathrm{NE}$ & + & $\mathrm{NE}$ & + \\
41 & S. Takoradi & chicken & - & - & - & + & + & - & + & - \\
42 & S. Wingrove & pigeon & $\mathrm{NE}$ & $\mathrm{NE}$ & $\mathrm{NE}$ & $\mathrm{NE}$ & - & $\mathrm{NE}$ & $\mathrm{NE}$ & $\mathrm{NE}$ \\
43 & S. Newport & chicken & - & - & - & + & + & + & - & + \\
44 & S.Larochelle & duck & - & - & - & + & $\mathrm{NE}$ & $\mathrm{NE}$ & $\mathrm{NE}$ & $\mathrm{NE}$ \\
45 & S. Vejle & turkey & - & + & + & + & + & + & + & + \\
46 & S. Tamale & chicken & - & - & - & $\mathrm{NE}$ & - & $\mathrm{NE}$ & - & $\mathrm{NE}$ \\
47 & S. Molade & chicken & + & - & + & $\mathrm{NE}$ & + & $\mathrm{NE}$ & - & + \\
48 & S. Kentucky & chicken & + & - & - & + & + & $\mathrm{NE}$ & $\mathrm{NE}$ & + \\
49 & S. Newport & chicken & - & - & - & + & + & + & $\mathrm{NE}$ & $\mathrm{NE}$ \\
50 & S. Inganda & duck & - & - & - & $\mathrm{NE}$ & + & + & + & + \\
51 & S. Kentucky & chicken & - & - & - & + & $\mathrm{NE}$ & $\mathrm{NE}$ & $\mathrm{NE}$ & $\mathrm{NE}$ \\
52 & S. Newport & chicken & - & - & - & + & + & + & + & $\mathrm{NE}$ \\
53 & S. Papuana & chicken & - & - & - & + & - & $\mathrm{NE}$ & - & $\mathrm{NE}$ \\
54 & S. Kentucky & chicken & - & - & - & + & + & + & - & + \\
55 & S. Santiago & chicken & - & - & + & $\mathrm{NE}$ & + & + & $\mathrm{NE}$ & $\mathrm{NE}$ \\
56 & S. Infantis & duck & - & - & - & - & - & - & - & + \\
57 & S. Angers & chicken & - & - & - & - & - & - & $\mathrm{NE}$ & - \\
58 & S. Bargny & chicken & - & - & + & + & + & + & $\mathrm{NE}$ & + \\
59 & S. Shubra & chicken & - & - & - & $\mathrm{NE}$ & $\mathrm{NE}$ & $\mathrm{NE}$ & $\mathrm{NE}$ & + \\
60 & S. Shangani & quail & - & - & - & - & - & - & $\mathrm{NE}$ & - \\
61 & S. Jedburgh & quail & $\mathrm{NE}$ & $\mathrm{NE}$ & $\mathrm{NE}$ & $\mathrm{NE}$ & $\mathrm{NE}$ & $\mathrm{NE}$ & $\mathrm{NE}$ & $\mathrm{NE}$ \\
62 & S. Jedburgh & quail & $\mathrm{NE}$ & $\mathrm{NE}$ & $\mathrm{NE}$ & $\mathrm{NE}$ & $\mathrm{NE}$ & $\mathrm{NE}$ & $\mathrm{NE}$ & $\mathrm{NE}$ \\
63 & Untyped & duck & $\mathrm{NE}$ & $\mathrm{NE}$ & $\mathrm{NE}$ & $\mathrm{NE}$ & $\mathrm{NE}$ & $\mathrm{NE}$ & $\mathrm{NE}$ & $\mathrm{NE}$ \\
Total examined & & 56 & 56 & 56 & 31 & 49 & 27 & 32 & 43 \\
Samples & & & & & & & & & \\
No. of positive & & 2 & 6 & 11 & 27 & 34 & 21 & 17 & 36 \\
$\%$ of positive & & 3.5 & 10.7 & 19.6 & 87 & 69.4 & 77.8 & 53.1 & 83.7 \\
\hline
\end{tabular}

(qepA - qnrS - aac $\left(6^{\prime}\right)$-ib-cr) $=$ resistant genes for quinolones

sul $1=$ resistant gene for sulfonamide \& bla TEM= resistant genes for B-lactamases

floR= resistant gene for florfenicol \& aadA2= resistant gene for streptomycin

tet $\mathrm{A}(\mathrm{A})=$ resistant gene for tetracycline. \&NE = not examined by $\mathrm{PCR}=$ sensitive samples.

Table 5. Nucleotide changes in the gyrA gene of Salmonella serovars.

\begin{tabular}{ccccccc}
\hline $\begin{array}{c}\text { Strain } \\
\text { no. }\end{array}$ & $\begin{array}{c}\text { Accession } \\
\text { no. }\end{array}$ & Serovar & Species & \multicolumn{3}{c}{ Nucleotide change at gyrA } \\
& & & & \multicolumn{3}{c}{ Positions } \\
\cline { 5 - 7 } & & & & TCC & GAC & GTA [Val] \\
& & & & {$[$ Ser] } & [Asp] & \\
\hline 14 & KP290114 & Infantis & Duck & None & None & None \\
40 & KP290113 & Enteritidis & Chicken & None & GGC & GCA [Ala] \\
& & & & & [Gly] & None \\
45 & KP290112 & Vejle & Turkey & None & None & None \\
& & & & & & \\
\hline
\end{tabular}


In turkey isolates, the highest percentage of resistance $(33.3 \%)$ was to Florphenicol, Nalidixic acid and Flumequin, Amoxicillin, Neomycin, Streptomycin, Oxytetracyclin, Sulfamethoxazole /Trimethoprim but other antimicrobial agents showed no resistance. These obtained result nearly in coordinated with Jodas and Hafez (2003) who reported that all of the examined Salmonella isolates from turkeys were sensitive to Enrofloxacin (98\%) while these results differ from Blackburn et al., (1982) who reported that all Salmonella strains isolated from turkeys were $(100 \%)$ resistant to Gentamicin. In addition to Pederson et al., (2002) who stated that $1.7 \%, 1.7 \%, 8.7 \%$, and $9 \%$ of strains isolated from Danish turkeys between 1995 and 2000, were resistant to Gentamicin,

Trimethoprim/

Sulphamethoxazole, Tetracycline and Streptomycin.

In quail isolates, $60 \%$ resistance to Florphenicol and the lowest percentage was $20 \%$ to Nalidixic acid, Flumequin, Oxytetracyclin and Sulfamethoxazole/ Trimethoprim while Ciprofloxacin, Enerofloxacin, Norfloxacin, Levofloxacin Cefotaxime, Ceftriaxone, Ceftazidime, Neomycin, Amikacin and Gentamycin showed sensitivity with $100 \%$ but Florphenicol was the lowest sensitivity with percentage of $40 \%$. The results in this study nearly in coordinated with Rahman et al., (2011) and differ from Bacci et al., (2012) who reported that the isolated Salmonellae from quails exhibited $89.2 \%$ resistance to Sulfamethoxazole and $24.3 \%$ to Ampicillin, Gentamicin, Sulfamethoxazole and Tetracycline in addition to Helm et al., (1999) who reported that Salmonella typhimurium isolated from bobwhite quail was resistant to Ampicillin, Chloramphenicol, Sulfonamides, and Tetracycline.

Pigeon isolates showed resistance (100\%) to Amoxicillin and Ampicilliln/ Sulbctam followed by Nalidix acid, Flumequin and Oxytetracyclin (50\%) while the isolates showed sensitivity with $100 \%$ to all examined antimicrobial agents except
Nalidixic acid, flumequin and oxytetracyclin that showed sensitivity with $50 \%$. These results agreed with Jahantigh and Nill (2010) who showed a high frequency of sensitivity to Ciprofloxacin, Norfloxacin and Gentamycin with percentage of $(100 \%)$ in Salmonella isolated from pigeon eggs but Banani et al., (2003) who found that all isolates of $S$. Enteritidis were sensitive to Ciprofloxacin, Ceftriaxone, and Florphenicol.

PCR was a perfect tool for accurate detection of Salmonella resistant genes and the results from qepA- qnrS- aac (6')-ib-cr, a resistant genes for quinolones were reported with a percentage of follow: (2/56 isolates) $3.5 \%$, (6 / 56 isolates) $10.7 \%$ and (11/ 56 isolates) $19.6 \%$ respectively. The results obtained for qepA gene nearly in coordinated with Lunn et al., (2010) who found that one isolate $(2.4 \%)$ showed the presence of qepA. While the results in this study differ from Hao et al., (2011) who reported aac (6')-Ib-cr and qnrS resistant genes in Salmonella with a percentage of $20.16 \%$ and $1.61 \%$ respectively. The sul 1 gene, a gene encoded for sulfonamide resistance was reported in the present study with a percentage of $87 \%$ ( 27 out of 31 isolates) these results differ from a study performed by Dessie et al., (2013) who identified sul2 in 33 Salmonella isolates but sull not identified in them in addition to another study performed by Zou et al., (2009) who detected sul1 in 11 of 16 isolates $(68.7 \%)$. The bla TEM gene, a gene encoded for B- lactam resistance was reported in the present study with a percentage of $69.4 \%$ (34 out of 49 isolates) these results differ from a study performed by Hur et al., (2011) who reported that $19 / 21$ penicillin resistant $S$. Enteritidis in Korea carried the bla (TEM) gene with a percentage of $(90.5 \%)$ but in another study done by Ahmed et al., (2009) the percentage of bla (TEM-1) was $10 \%$ which was identified in between 10 Salmonella isolates from retail chicken meat in Hiroshima, Japan. The floR gene, a gene encoded for florphenicol resistance 
was reported in the present study with a percentage of $77.8 \%$ (21/ 27 isolates) these results nearly in accordance with $\mathrm{Lu}$ et al., (2014) who reported that 108 S. Indiana possessed floR gene with a percentage of 81.2\%. However, Cloeckaert et al., (2000) reported that Salmonella enterica serovar Agona strains isolated from poultry harbor floR gene, conferring crossresistance to Chloramphenicol and Florphenicol. The aadA2 gene, a gene encoded for Streptomycin resistance was reported in the current study with a percentage of $53.1 \%$ (17/ 32 isolates) these results came in contrary with a study performed by Shahada et al., (2006) who reported that all streptomycin resistant $S$. Infantis from poultry in Japan carried aadA1 gene while Sheng et al., (2004) who reported aadA2 gene in three Salmonella isolates from seventy-three isolates from retail meats. The tet $\mathrm{A}$ gene, a gene encoded for tetracycline resistance was reported in the present study with a percentage of $83.7 \%$ (36/43 isolates) these results nearly in coordinated with Lu et al., (2014) who reported that 108 S. Indiana possessed tet A gene with a percentage of $81.2 \%$ and Shahada et al., (2006) who reported that $89 \%$ of Oxytetracyclineresistant $S$. Infantis from poultry in Japan carried the tet (A) gene while these results differ from a study performed by Ahmed et al., (2012) who identified tetracycline resistance gene tet A in 14 out of 21 (66.7\%) Salmonella isolates.

Mutations in the gyrA gene were known to be the main resistance mechanism to quinolones. However, there are other mechanisms as the plasmid mediated resistance and this was obvious in the current gyrA sequence results. S. Enteritidis showed double mutations in the codons Asp87 and Val176 while $S$. Infantis and $S$. Vejle showed no mutations at gyrA gene. The results in this study nearly in coordinated with Reyna et al., (1995) who stated that double and triple combinatorial mutations involving residues 83 and 87 of gyrA are associated with much higher resistance levels than those of single mutations and Hirose et al., (2002) who reported a single mutation in the gyrA gene, at either position 83 or 87 of gyrA in Salmonella enterica Serovar Typhi and Serovar Paratyphi A and double mutations in the gyrA gene at both position 83 and position 87 of gyrA in strains with highlevel resistance to Fluoroquinolones induced by in vitro selection with ciprofloxacin. The mutations (Ser83Phe and/or Asp87Gly) were previously recorded in many reference Salmonella strains and also in strains isolated from food and domestic or wild birds as reported by (Reyna et al., 1995; Hirose et al., 2002 and De Souza et al., 2011).

Because of much higher resistances of Salmonella to different antimicrobials that reported in this study using sensitivity tests, from PCR and sequencing techniques, it's recommended not to apply any antimicrobial agents for Salmonella treatment without application of sensitivity tests to avoid these resistance which have adverse effect on poultry industry. Also peoples shouldn't eat under cooked poultry and poultry products to avoid infection with such resistant Salmonellae.

\section{REFERENCES}

Ahmed, A.M., Shimabukuro, H., Shimamoto, T. 2009. Isolation and molecular characterization of multidrugresistant strains of Escherichia coli and Salmonella from retail chicken meat in Japan. J. Food Sci., 74(7):M 405-410.

Ahmed, A. M., Shimamoto, T. 2012. Genetic analysis of multiple antimicrobial resistance in Salmonella isolated from diseased broilers in Egypt. Microbiology and Immunology 56(4):254-261.

Ang, J.Y., Ezike, E., Asmar, B.I. 2004. Antibacterial resistance. Indian Journal of Pediatrics 71:229-239.

Bacci, C., Boni, E., Alpigiani, I., Lanzoni, E., Bonardi, S., Brindani, F. 2012. Phenotypicand genotypic features of 
antibiotic resistance in Salmonella enterica isolated from chicken meat and chicken and quail carcasses. Int. J. Food Microbiol. 160(1):16-23.

Banani, M., Pourbakhsh, S. A., Khaki, P., Nikookhesal, G. H. 2003. Serotyping and drug sensitivity of Salmonella isolates from commercial chickens and domestic pigeons submitted to Razi institute. Pajouhesh-va-Sazandegi. In Animal Sciences 59:92-99.

Blackburn, O.B., Sclater, L.K., Swanson, M.R. 1982. Antibiotic resistance of members of THA genus Salmonella isolated from chicken, turkeys, cattle, and swine in the United States during October 1981 through September. Am. J.Vet. Res. 45:1245-1249.

Boris H., Borka, S., Gordan, K., Fani, K. 2012. Antimicrobial resistance and serotyping of Salmonella enterica subsp. enterica isolated from poultry in Croatia. Vet. Arhiv. 82:371-381.

Cardoso, M.O., Ribeiro, A.R., Santos, L.R., Pilotto, F., Moraes, H.S., Salle, C.P., Rocha, S.S., Nascimento, V.P. 2006. Antibiotic resistance of Salmonella Enteritidis isolated from broiler carcasses. Braz. J. Microbiol., 37:368371.

Cattoir, V., Poirel, L., Nordmann, P. 2008. plasmid - mediated quinolone resistance pump QepA2 in an Escherichia coli isolated from France. Antimicrob. Agents Chemother., 52:3801- 3804.

Cloeckaert, A., Sidi, B. K., Flaujac, G., Imberechts, H., D'Hooghe, I., Chaslus-Dancla, E. 2000. Occurrence of Salmonella enterica serovar Typhimurium DT104-like antibiotic resistance gene cluster including the floR gene in S. enterica serovar Agona. Antimicrob Agents Chemother. ; 44(5):1359-1361.

CLSI (2011): Clinical and Laboratory Standards Institute antimicrobial susceptibility testing standards M2- A9 and M7-A7.

Colom, K., Perez, J., Alonoso, R., Fernandez- Arangguiz, A., Larino, E.,
Cisterna, R. 2003. simple and reliable multiplex PCR assay for detection of bla TEM, bla SHV, bla OXA-1 genes in Enterobacteriaceae.

FEMS Microbiology Letters 223:147-151.

Cruchaga, S., Echieta, A., Aladuena, A., Rena, J.G., N., Usera, M.A. 2001. Antimicrobial resistance in Salmonella from human food and animals in Spain in 1998.J. Antimicrob. Chemother., 47:315 321.

Dessie, H.K., Bae, D.H., Lee, Y.J. 2013. Characterization of integrons and their cassettes in Escherichia coli and Salmonella isolates from poultry in Korea. Poult Sci.; 92(11):3036-3043.

De Souza, R.B., Magnani, M., Ferrari, R.G., Kottwitz, L.B.M., Sartori, D., Tognim, M.C.B., de Oliveira, T.C.R. 2011. Detection of quinolone-resistance mutations in Salmonella strains of epidemic and poultry origin. Brazilian Journal of Microbiology. 42:211-215.

Doublet, B., Lailler, R., Meunier, D., Brisabois, A., Boyd, D., Mulvey, M. R., Chaslus- Dancla, E. and Cloeckaert, A. 2003. Salmonella entrica serovar Albany. Emerging Infectious Diseases. 9 (5):585-591.

Fàbrega, A., Madurga, S., Giralt, E., Vila, J. 2009. Mechanism of action of and resistance to quinolones. Microbial Biotechnology 2:40-61.

Finegold, S. M., Martin, E. T. 1982. Diagnostic microbiology. $6^{\text {th }}$ Ed., The C.V. Mosby Company, St. Louis, Toronto, London.

Hao, H., Yang, B., Shi, J., Xi, M., Wang, X., Cui, Y., Meng, J. 2011. drug resistance and related genes of chicken borne Salmonella to quinolone and fluoroquinolones. Wei Sheng Wu Xue Bao. 51(10):1413-1420.

Hegazy, A.A. 1991. studies on Salmonella infections in ducks. M.V.Sc.Thesis Fac. Med. Alex. Univ.

Helm, J.D., Hines, R. K., Hill, J.E., Caver, J.A. 1999. Multiple drugresistant Salmonella typhimurium DT104 and DT104b isolated in 
bobwhite quail (Colinus virginianus). Avian Dis. 43(4):788-791.

Hirose, K., Hashimoto, A., Tamura, K., Kawamura, Y., Ezaki, T., Sagara, H., Watanabe, H. 2002. DNA Sequence Analysis of DNA Gyrase and DNA Topoisomerase IV Quinolone Resistance-Determining Regions of Salmonella enteric Serovar Typhi and Serovar Paratyphi A. antimicrobial agents and chemotherapy, 46(10):32493252.

Hur, J., Kim, J.H., Park, J.H., Lee, Y.J., Lee, J.H. 2011. Molecular andvirulence characteristics of multidrug resistant Salmonella Enteritidis strains isolated from poultry. Vet J. 189(3):306-311.

Jahantigh, M., Nili, H. 2010. Drug resistance of Salmonella spp. isolated from pigeon eggs. Comparative Clinical Pathology; 19(4): 437-439.

Jodas, S., Hafez, H. M. 2003. Surveillance on antimicrobial resistance in Escherichia coli and Salmonella isolates from poultry between 1993 and 1999. Archiv fur Geflugelkunde; 67(1):16-21.

Khan, M., Suryanarayan, P., Ahmed, M., Vaswani, R., Faheem, S. 2010. Antimicrobial susceptibility of Salmonella isolates from chicken meat samples in Dubai, United Arab Emirates. International Journal of Food, Nutrition and Public Health, 3(2):149-159.

Lu, Y., Zhao, H., Liu, Y., Zhou, X., Beier, R.C., Wu, G., Hou, X. 2014. Characterization of Multidrug-Resistant Salmonella enterica Serovars Indiana and Enteritidis from Chickens in Eastern China. PLoS. 9(5):96050.

Lunn, A. D., fabrega, A., SanchezCespedes, J., Vila, J. 2010. Prevalence of mechanisms decreasing quinolonesusceptibility among Salmonella spp. Clinical isolates. International Microbiology, 13:15-20.

Munawwar, A., Priyanka, S., Mohammed, M., Reshma, B. and Sultan, M. 2010. Robicsek, A., Strahilevitz, J., Sahm, D. F., Jacoby, G. A., Hooper, D.C. 2006. qnr
Antimicrobial susceptibility of Salmonella isolates from chicken meat samples in Dubai, United Arab Emirates. International Journal of Food, Nutrition and Public Health, 3(2):10-20.

Pan, Z., Wang, X., Zhang, X., Geng, S., Chen, X., Pan, W., Cong, Q., Liu, X., Jiao, X., Liu, X. 2009. Changes in antimicrobial resistance among Salmonella enterica subspecies enterica serovar Pullorum isolates in China from 1962 to 2007. Vet Microbiol. 136(34):387-392.

Pederson, K., Hansen, H.C., Jorgensen, J. C., Borck B. 2002. Serovars of Salmonella isolated from Danish turkeys between 1995 and 2000 and their antibiotic resistance. Vet. Rec. 150:471474.

Rahman, M. M., Hossain, M. K., Akhter, M. R., Hasan, S. M. K., Rahman, M. M. 2011. Characterization and antibiogram study of salmonella serovars isolated from duck, quail and pigeon in Dinajpur district of Bangladesh. International Journal of Sustainable Agricultural Technology 7(2):23-29.

Rahman, H., Mukit, A., Goswami, S., Kabita, R. 1999. Outbreak of Salmonellosis in ducklings in Assam. Indian Journal of Comparative Microbiology, Immunology and Infectious Diseases. 20(1):26-28.

Randall, L.P., Cooles, S. W., Osborn, M. K., Piddock, L. J. V., Woodward, M. J. 2004. Antibiotic resistance genes, integrons and multiple antibiotic resistance in thirty- five serotypes of Salmonella enterica isolated from humans and animals in the UK. Journal of Antimicrobial Chemotherapy. 53:208216.

Reyna, F., Huesca, M., González, V., Fuchs, L.Y. $1995 . \quad$ Salmonella Typhimurium gyrA Mutations Associated with Fluoroquinolone Resistance. Antimicrobial agents and chemotherapy, 39(7):1621-1623.

prevalence in ceftazidime- resistant Enterobacteriaceae isolates from the 
USA. Antimicrob Agents Chemother 50: 2872- 2874.

Sabarinath, A., Tiwari, K. P., Deallie, C. Belot, G., Vanpee, G., Matthew, V., Sharma, R., Hariharan, H. 2011. Antimicrobial resistance and phylogenetic Groups of Commensal Escherichia coli isolates from healthy Pigs in Grenada. Available from: www. webmedcentral.com.

Shahada, F., Chuma, T., Tobata, T., Okamoto, K., Sueyoshi, M., Takase, K. 2006. Molecular epidemiology of antimicrobial resistance among Salmonella enterica serovar Infantis from poultry in Kagoshima, Japan. Int $\mathrm{J}$ Antimicrob Agents. ; 28(4):302-307.

Sheng, C., Shaohua, Z., David, G. W., Carl, M., Ran L., Hanchun, Y., Patrick, F., McDermott, S., Jianghong, M. 2004. Characterization of multiple antibiotic resistant Salmonella serovars Isolated from Retail Meats Appl. Environ. Microbiol. 70(1):1-7.

Snow, L.C., Davies, R.H., Christiansen, K.H., Carrique-Mas, J.J., Cook, A.J.,

Evans, S.J. 2007. Survey of Salmonella prevalence on commercial turkey breeding and fattening farms in the UK in 2006 to 2007. Vet Rec. 169(19):493.

Walker, R. A., Lindsay, E. and Woodward, M. J. 2001. Variation in clonality and antibiotic- resistance genes among multi- resistant Salmonella enterica serotype Typhimurium phage- type U302 (MR U302) from humans, animals and foods. Microbiological Research 7: 13- 21.

WHO. 2006. Drug resistant Salmonella- fact sheet No. 139 Revised April (2005).

Zdragas, A., Mazaraki, K., Vafeas, G., Giantzi, V., Papadopoulos, T., Ekateriniadou, L. 2012. Prevalence, seasonal occurrence and antimicrobial resistance of Salmonella in poultry retail products in Greece. Letters in Applied Microbiology. 55(4):308-313.

Zou, W., Frye, J. G., Chang, C.-W., Liu, J., Cerniglia, C. E., Nayak, R. 2009. Microarray analysis of antimicrobial resistance genes in Salmonella enterica from preharvest poultry environment. Journal of Applied Microbiology. 107(3):906-914. 